\title{
Reducing cutoff effects in maximally twisted LQCD close to the chiral limit
}

\author{
R. Frezzotti ${ }^{a}$, G. Martinelli ${ }^{b}$, M. Papinutto ${ }^{c}$, G.C. Rossid ${ }^{d, c}$ \\ ${ }^{a}$ INFN, Sezione di Milano \\ and Dipartimento di Fisica, Università di Milano "Bicocca" \\ Piazza della Scienza 3 - 20126 Milano, Italy \\ ${ }^{b}$ Dipartimento di Fisica, Università di Roma "La Sapienza" \\ and INFN, Sezione di Roma "La Sapienza" \\ Piazzale A. Moro - 00185 Roma, Italy \\ ${ }^{c}$ John von Neumann-Institut für Computing NIC \\ Platanenallee 6, D-15738 Zeuthen, Germany \\ d Dipartimento di Fisica, Università di Roma "Tor Vergata" \\ and INFN, Sezione di Roma 2 \\ Via della Ricerca Scientifica - 00133 Roma, Italy
}

\begin{abstract}
When analyzed in terms of the Symanzik expansion, lattice correlators of multi-local (gauge-invariant) operators with non-trivial continuum limit exhibit in maximally twisted lattice QCD "infrared divergent" cutoff effects of the type $a^{2 k} /\left(m_{\pi}^{2}\right)^{h}, 2 k \geq h \geq 1$ ( $k, h$ integers), which tend to become numerically large when the pion mass gets small. We prove that, if the action is $\mathrm{O}(a)$ improved à la Symanzik or, alternatively, the critical mass counter-term is chosen in some "optimal" way, these lattice artifacts are reduced to terms that are at worst of the order $a^{2}\left(a^{2} / m_{\pi}^{2}\right)^{k-1}, k \geq 1$. This implies that the continuum extrapolation of lattice results is smooth at least down to values of the quark mass, $m_{q}$, satisfying the order of magnitude inequality $m_{q}>a^{2} \Lambda_{\mathrm{QCD}}^{3}$.
\end{abstract}




\section{Introduction and main results}

Although in lattice QCD at maximal twist (Mtm-LQCD) $\mathrm{O}(a)$ discretization effects (actually all $\mathrm{O}\left(a^{2 k+1}\right), k \geq 0$ effects) are absent or easily eliminated [1, 2, 3], it turns out that, because of the explicit breaking of parity and isospin symmetry present in the action, correlators are affected by dangerous artifacts of relative order $a^{2 k}, k \geq 1$, which are enhanced by inverse powers of the (squared) pion mass, as the latter becomes small. Terms of this kind may have been seen in the simulations reported in ref. 44.

When analyzed in terms of the Symanzik expansion, the expectation values of multi-local (gauge-invariant) operators, $\left.\langle O\rangle\right|_{m_{q}} ^{L}$, exhibit, as $m_{\pi}^{2} \rightarrow 0$, what we will call "infrared (IR) divergent" cutoff effects with a behaviour of the form ${ }^{1}$

$$
\left.\langle O\rangle\right|_{m_{q}} ^{L}=\left.\langle O\rangle\right|_{m_{q}} ^{\mathrm{cont}}\left[1+\mathrm{O}\left(\frac{a^{2 k}}{\left(m_{\pi}^{2}\right)^{h}}\right)\right], \quad 2 k \geq h \geq 1(k, h \text { integers }),
$$

where we have assumed that the lattice correlator has a non-trivial continuum limit.

It is important to remark that the existence of pion poles in the Symanzik expansion of a lattice correlator in no way means that the latter diverges as $m_{q} \rightarrow 0$, but only that the Symanzik expansion may break down if the order of magnitude inequality $m_{\pi}^{2}>a$ is violated [5].

We will prove that, if the action is $\mathrm{O}(a)$ improved $\grave{a}$ la Symanzik by introducing the lattice clover term [6] (with its non-perturbatively determined $c_{S W}$ coefficient [7), or the critical mass counter-term is chosen in some "optimal" way, these artifacts will be reduced to terms that are at worst of order $a^{2}\left(a^{2} / m_{\pi}^{2}\right)^{k-1}, k \geq 1$.

The idea that a suitable definition of critical mass exists which can lead to a smoothing out of chirally enhanced lattice artifacts or perhaps be of help in getting improvement was already put forward in the context of chiral perturbation theory $(\chi \mathrm{PT})$ in refs. [8] and 9], respectively.

A crucial consequence of the analysis we present in this paper is that the strong (order of magnitude) inequality

$$
m_{q}>a \Lambda_{\mathrm{QCD}}^{2},
$$

invoked in ref. 2] in order to have the phase of the chiral vacuum driven by the quark mass term and not by the (twisted) Wilson term, can be relaxed to the weaker relation

$$
m_{q}>a^{2} \Lambda_{\mathrm{QCD}}^{3}
$$

\footnotetext{
${ }^{1}$ Often for short we do not include powers of $\Lambda_{\mathrm{QCD}}$ required to match physical dimensions.
} 
before large cutoff effects are possibly met when the quark mass is lowered at fixed $a$. The bound (1.3) is fairly weak as it allows simulations in a region of quark masses that correspond to rather light pions (with masses close to around $200 \mathrm{MeV}$ for typical present-day lattice spacings).

In order to make this observation useful in practice, it is however necessary to have a handle on the numerical coefficients entering the bound (1.3). We will argue that one can monitor the "safe" region of quark masses by measuring the mass of the charged pion as a function of $m_{q}$. It can be shown, in fact, that, once the leading "IR divergent" cutoff effects have been eliminated (and under the assumption that chiral symmetry is spontaneously broken in the continuum), the lattice squared mass of the charged pion, for sufficiently small $m_{q}$, is a linear function of $m_{q}$ (with only small $\mathrm{O}\left(a^{4}\right)$ and $\mathrm{O}\left(m_{q}^{2}\right)$ distortions) at least until the pion mass remains within the bound hinted at by eq. (1.3).

The situation one finds in this kinematic regime is thus reminiscent of the ideal continuum case, where the Gell-Mann-Oakes-Renner (GMOR) relation [10] ensures the proportionality of $m_{\pi}^{2}$ to the mass of the quark. Armed with the lattice analog of the GMOR relation one can exploit the previous considerations to argue that, vice-versa, at fixed $a, m_{q}$ should not be decreased beyond the point where non-linearities show up.

The effectiveness of Mtm-LQCD in removing $\mathrm{O}(a)$ discretization errors was successfully tested in quenched simulations in refs. [11]. The ability of the optimal choice of the critical mass in diminishing the magnitude of lattice artifacts at small quark mass has been beautifully demonstrated in the recent works of refs. [12, 13, 14. Results of unquenched simulations [15], though encouraging, are still rather preliminary due to the complicated phase structure [16] of Wilson fermions when $m_{q} / \Lambda_{\mathrm{QCD}}$ is numerically comparable to (or smaller than) $a^{2} \Lambda_{\mathrm{QCD}}^{2}$.

The plan of the paper is as follows. In Section 2 we analyze the form of the Symanzik expansion of lattice correlators beyond $\mathrm{O}(a)$ and explain why and how "IR divergent" cutoff effects arise in this context. In Section 3 we discuss two ways of removing all the leading "IR divergent" cutoff effects and we describe the structure of the left-over "IR divergent" terms. In Section 4 we derive the lattice GMOR relation and we discuss how it can be used to monitor the lowest value of the quark mass that can be safely employed if the lattice spacing is held fixed. Finally in Section 5 we collect some remarks on the peculiar structure of the lattice artifacts affecting lattice hadron energies and the pion decay constant. Conclusions can be found in Section 6. In an Appendix we prove that automatic $\mathrm{O}\left(a^{2 k+1}\right)$ improvement of the lattice expectation values of parity-even operators holds in Mtm-LQCD, by using an argument which does not rely on the spurionic transformation $r \rightarrow-r$, 
hence on the $r$-parity properties of the critical mass.

\section{Symanzik analysis of "IR divergent" cutoff artifacts}

The expression of fermionic action of Mtm-LQCD is given in the physical quark basis [2, 3] by the formula

$$
S_{\mathrm{tmF}}^{L}=a^{4} \sum_{x} \bar{\psi}^{L}(x)\left[\gamma \cdot \widetilde{\nabla}+m_{q}-i \gamma_{5} \tau_{3}\left(-a \frac{r}{2} \nabla^{*} \cdot \nabla+M_{\mathrm{cr}}^{e}(r)\right)\right] \psi^{L}(x),
$$

where $\psi^{L}$ is a lattice fermion doublet, $r$ is the Wilson parameter and $m_{q}$ is the bare quark mass, i.e. the real parameter which in the continuum will provide a non-vanishing mass to the pion. $M_{\mathrm{cr}}^{e}$ is any sensible (from the point of view of renormalization theory) "estimate" of the critical mass which, in order to match the $r$-parity properties of the Wilson term, should be taken as an odd function of $r$ [2].

The study of cutoff artifacts affecting lattice correlators in Mtm-LQCD can be elegantly made in the language of the Symanzik expansion. A full analysis of cutoff effects beyond $\mathrm{O}(a)$ is of course extremely complicated. Fortunately it is not necessary, if we limit the discussion to the terms that are most strongly enhanced as the quark mass is decreased. This analysis will be carried out in the next section.

For completeness we show in an Appendix that automatic $\mathrm{O}(a)$ (actually $\left.\mathrm{O}\left(a^{2 k+1}\right), k \geq 0\right)$ improvement directly follows from the symmetry of the lattice theory under the transformation $P \times \mathcal{D}_{d} \times\left(m_{q} \rightarrow-m_{q}\right)$, where $P$ is parity and the transformation $\mathcal{D}_{d} \times\left(m_{q} \rightarrow-m_{q}\right)$ counts the parity of the overall dimension of an operator. The explicit definition of the parity operation $P$ and the transformation $\mathcal{D}_{d}$ can be found in eqs. (A.1) and (A.2). The proof we give does not involve any change of sign of the Wilson parameter, thus it is independent of the $r$-parity properties of the critical mass ${ }^{2}$, unlike the argument developed in ref. [2].

\subsection{The Symanzik LEEA of Mtm-LQCD}

With reference to the fermionic lattice action (2.1), the low energy effective action (LEEA) of Mtm-LQCD, $S_{\text {Sym }}$, can be conveniently written in the form

$$
S_{\mathrm{Sym}}=\int d^{4} y\left[\mathcal{L}_{4}(y)+\sum_{k=0}^{\infty} a^{2 k+1} \ell_{4+2 k+1}(y)+\sum_{k=1}^{\infty} a^{2 k} \ell_{4+2 k}(y)\right],
$$

\footnotetext{
${ }^{2}$ We wish to thank M. Lüscher for a stimulating discussion on this issue during the "Twisted Mass Lattice Fermions" workshop held in Villa Mondragone (Frascati - Italy) on March 14-15, 2005.
} 
where $\mathcal{L}_{4}=\frac{1}{2 g_{0}^{2}} \operatorname{tr}(F \cdot F)+\bar{\psi}\left(\gamma \cdot D+m_{q}\right) \psi$ is the target continuum QCD Lagrangian density. A number of interesting properties enjoyed by the above LEEA can be proved which we summarize below.

- Lagrangian density terms of even dimension, $\ell_{2 k}$, in eq. (2.2) are parityeven, while terms of odd dimension, $\ell_{2 k+1}$, are parity-odd and twisted in iso-spin space. Thus the latter have the quantum numbers of the neutral pion. These parity properties follow from dimensional arguments and the invariance of the LEEA of Mtm-LQCD under the transformation $P \times \mathcal{D}_{d} \times$ $\left(m_{q} \rightarrow-m_{q}\right)$, inherited from the correspondent invariance of the lattice theory (see Appendix).

- The term of order $a$ in eq. (2.2),$\ell_{5}$, is given (after use of the equations of motion of continuum QCD) by the linear combination

$$
\begin{aligned}
& \ell_{5}=\delta_{5, S W} \ell_{5, S W}+\delta_{5, m^{2}} \ell_{5, m^{2}}+\delta_{5, e} \ell_{5, e}, \\
& \ell_{5, S W}=\frac{i}{4} \bar{\psi}[\sigma \cdot F] i \gamma_{5} \tau_{3} \psi, \quad \ell_{5, m^{2}}=m_{q}^{2} \bar{\psi} i \gamma_{5} \tau_{3} \psi, \quad \ell_{5, e}=\Lambda_{\mathrm{QCD}}^{2} \bar{\psi} i \gamma_{5} \tau_{3} \psi,
\end{aligned}
$$

where the coefficients $\delta_{5, S W}, \delta_{5, m^{2}}$ and $\delta_{5, e}$ are $\mathrm{O}(1)$ dimensionless quantities, odd in $r$. The operator $\ell_{5, e}$ arises from the need to describe order $a$ uncertainties entering any non-perturbative determination of the critical mass and goes together with $\ell_{5, S W}$. Both $\ell_{5, S W}$ and $\ell_{5, e}$ could be made to disappear from eq. (2.2) by introducing in the Mtm-LQCD action the SW (clover)-term with the appropriate non-perturbatively determined $c_{S W}$ coefficient and at the same time setting the critical mass to its correspondingly $\mathrm{O}(a)$ improved value.

- Higher order ambiguities $(k \geq 1)$ in the critical mass will be described by terms proportional to odd powers of $a$, more precisely of the kind

$$
a^{2 k+1} \delta_{4+2 k+1, e} \ell_{4+2 k+1, e}=a^{2 k+1} \delta_{4+2 k+1, e}\left(\Lambda_{\mathrm{QCD}}\right)^{2 k+2} \bar{\psi} i \gamma_{5} \tau_{3} \psi \text {. }
$$

The structure of these terms, which will all contribute to $\mathcal{L}_{\text {odd }}$ (see eq. (2.8) below), follows again from obvious dimensional arguments plus the invariance of the lattice action under the transformation $P \times \mathcal{D}_{d} \times\left(m_{q} \rightarrow-m_{q}\right)$.

\subsection{Describing Mtm-LQCD correlators beyond $\mathrm{O}(a)$}

We are interested in the Symanzik description of the lattice artifacts affecting connected expectation values of $n$-point, multi-local, multiplicative renormalizable (m.r.) and gauge-invariant operators

$$
O\left(x_{1}, x_{2}, \ldots, x_{n}\right)=\prod_{j=1}^{n} O_{j}\left(x_{j}\right) \equiv O(x), \quad x_{1} \neq x_{2} \neq \ldots \neq x_{n}
$$


which we take to have continuum vacuum quantum numbers, so as to yield a result that does not trivially vanish as $a \rightarrow 0$. In particular, in order to ensure automatic $\mathrm{O}(a)$ improvement [2] we will assume that $O$ is parity invariant in which case its Symanzik expansion will contain only even powers of $a$. Schematically we write

$$
\left.\langle O(x)\rangle\right|_{m_{q}} ^{L}=\left.\left\langle\left[O(x)+\Delta_{\text {odd }} O(x)+\Delta_{\text {even }} O(x)\right] e^{-\int d^{4} y\left[\mathcal{L}_{\text {odd }}(y)+\mathcal{L}_{\text {even }}(y)\right]}\right\rangle\right|_{m_{q}} ^{\text {cont }},
$$

where for short we have introduced the compact notations

$$
\mathcal{L}_{\text {odd }}=\sum_{k=0}^{\infty} a^{2 k+1} \ell_{4+2 k+1}, \quad \mathcal{L}_{\text {even }}=\sum_{k=1}^{\infty} a^{2 k} \ell_{4+2 k} .
$$

The operators $\Delta_{\text {odd }} O$ (resp. $\Delta_{\text {even }} O$ ) have their origin in the need of regularizing terms where a parity-odd (resp. parity-even) product of $\mathcal{L}_{\text {odd }}$ and/or $\mathcal{L}_{\text {even }}$ insertions comes in contact with some of the points where the local operator factors appearing in $O$ are concentrated. $\Delta_{\text {odd }} O$ (resp. $\Delta_{\text {even }} O$ ) counter-terms have an expansion in odd (resp. even) powers of $a$. We recall that they can be viewed as the $n$-point operators necessary for the on-shell improvement of $O$ [17, 7].

For the purpose of this discussion we imagine expanding the r.h.s. of eq. (2.7) in powers of $\int d^{4} y \mathcal{L}_{\text {odd }}(y)$ and/or $\int d^{4} y \mathcal{L}_{\text {even }}(y)$. Terms with $j$ and/or $j^{\prime}$ insertions of the first and/or the second of these factors will generate in the Symanzik expansion $h$-fold $1 / m_{\pi}^{2}$ pion poles with $1 \leq h \leq j+j^{\prime}$.

\subsection{Pion poles and "IR divergent" cutoff effects}

Although a complete analysis of all the "IR divergent" cutoff effects is very complicated, the structure of the leading ones $(h=2 k$ in eq. (1.1) $)$ is rather simple, as they only come from continuum correlators where $2 k$ factors $\int d^{4} y \mathcal{L}_{\text {odd }}(y)$ are inserted. More precisely the leading "IR divergent" cutoff effects are identified on the basis of the following

Result: In the Symanzik expansion of $\left.\langle O(x)\rangle\right|_{m_{q}} ^{L}$ at order $a^{2 k}(k \geq 1)$ there appear terms with a $2 k$-fold pion pole and residues proportional to $\left|\left\langle\Omega\left|\mathcal{L}_{\text {odd }}\right| \pi^{0}(\mathbf{0})\right\rangle\right|^{2 k}$, where $\langle\Omega|$ and $\left|\pi^{0}(\mathbf{0})\right\rangle$ denote the vacuum and the one$\pi^{0}$ state at zero three-momentum, respectively. Putting different factors together, each one of these terms can be seen to be schematically of the form $\left(\right.$ recall $\left.\mathcal{L}_{\text {odd }}=\mathrm{O}(a)\right)$

$$
\left[\left(\frac{1}{m_{\pi}^{2}}\right)^{2 k}\left(\xi_{\pi}\left(m_{q}\right)\right)^{2 k} \mathcal{M}\left[O ;\left\{\pi^{0}(\mathbf{0})\right\}_{2 k}\right]\right]_{m_{q}}^{\mathrm{cont}}
$$


where we have generically denoted by $\mathcal{M}\left[O ;\left\{\pi^{0}(\mathbf{0})\right\}_{2 k}\right]$ the $2 k$-particle matrix elements of the operator $O$, with each of the $2 k$ particles being a neutral pion at zero three-momentum and we have introduced the short-hand notation

$$
\xi_{\pi}\left(m_{q}\right)=\left|\left\langle\Omega\left|\mathcal{L}_{\text {odd }}\right| \pi^{0}(\mathbf{0})\right\rangle\right|_{m_{q}}^{\text {cont }} .
$$

The proof of the above Result can be obtained on the basis of 1) the general theorems of quantum field theory governing the appearance of poles in correlators [18, 2) the notion of "partially disconnected" diagrams which come about when reducing pairs of pions from in and out states of a multipion matrix element, 3$)$ the observation that the $(2 k+1)$-pion matrix elements of $\mathcal{L}_{\text {odd }}$ can all be shown to be proportional to $\xi_{\pi}$ in the chiral limit, through a repeated use of soft pion theorems [19], in which pions are successively reduced out.

Less "IR divergent" cutoff effects (those with $h$ strictly smaller than $2 k$ in eq. (1.1) ) come either from terms with insertions of $\int d^{4} y \mathcal{L}_{\text {even }}(y)$ and/or pairs of $\int d^{4} y \mathcal{L}_{\text {odd }}(y)$ factors, or from contributions of more complicated intermediate states other than straight zero three-momentum pions or from both. In all cases one gets terms with extra $a^{2}$ powers not "accompanied" by an equal number of $1 /\left(m_{\pi}^{2}\right)^{2}$ factors.

\section{Reducing "IR divergent" cutoff artifacts}

We have shown in the previous sections that close to the chiral limit the most "IR divergent" discretization artifacts affecting Mtm-LQCD correlators at order $a^{2 k}$ are proportional to $2 k$ powers of $\xi_{\pi}$ (see eq. (2.9)). Since $\mathcal{L}_{\text {odd }}=a \ell_{5}+\mathrm{O}\left(a^{3}\right)$, this also means that at leading order in $a$ each multiple pion pole residue is proportional to $\left|\left\langle\Omega\left|\ell_{5}\right| \pi^{0}(\mathbf{0})\right\rangle\right|^{2 k}$. It is an immediate conclusion of this analysis that all these dangerous cutoff effects can be removed from lattice data if we can either eliminate $\ell_{5}$ from the Symanzik LEEA of Mtm-LQCD or set $\xi_{\pi}$ to zero. Actually, in the last case a somewhat weaker condition is sufficient. Indeed, we will see that it is enough to reduce $\xi_{\pi}$ to a quantity of order $a m_{\pi}^{2}$.

\subsection{Improving the Mtm-LQCD action by the SW-term}

The obvious, field-theoretical way to eliminate $\ell_{5}$ from the Symanzik LEEA of Mtm-LQCD consists in making use of the $\mathrm{O}(a)$ improved action

$$
S_{\mathrm{tmF}}^{I L}=a^{4} \sum_{x} \bar{\psi}^{L}(x)\left[\gamma \cdot \widetilde{\nabla}+m_{q}+\right.
$$




$$
\left.-i \gamma_{5} \tau_{3}\left(-a \frac{r}{2} \nabla^{*} \cdot \nabla+M_{\mathrm{cr}}^{I e}(r)+\frac{i}{4} c_{S W}(r)[\sigma \cdot F]^{L}\right)\right] \psi^{L}(x),
$$

where $c_{S W}$ is fixed in the appropriate non-perturbative way [7] and $M_{c r}^{I e}$ is an improved estimate of the critical mass.

In this situation the lattice correlation functions of the theory will admit a Symanzik description in terms of a LEEA where the operators $\ell_{5, S W}$ and $\ell_{5, e}$ are absent, and $\ell_{5}$ will be simply given by $\ell_{5, m^{2}}$ (see eq. (2.3)). The left-over contributions arising from the insertions of $\ell_{5, m^{2}}$ in $\left.\langle O\rangle\right|_{m_{q}} ^{\text {cont will yield terms }}$ that are at most of order $\left(a m_{q}^{2} / m_{\pi}^{2}\right)^{2 k} \simeq\left(a m_{q}\right)^{2 k}$, hence negligible in the chiral limit. It is instead the next odd operator in the Symanzik expansion, $a^{3} \ell_{7}$, which comes into play.

A detailed combinatoric analysis based on the structure of the non-leading "IR divergent" cutoff effects reveals that the worst lattice artifacts left behind in correlators after the clover cure are of the kind $a^{2}\left(a^{2} / m_{\pi}^{2}\right)^{k-1}, k \geq 1$.

\subsection{Optimal choice of the critical mass}

The alternative strategy to eliminate all the leading "IR divergent" cutoff effects consists in leaving the Mtm-LQCD action of the form (2.1), but fixing the critical mass through the condition

$$
\lim _{m_{q} \rightarrow 0^{+}} \xi_{\pi}\left(m_{q}\right)=\lim _{m_{q} \rightarrow 0^{+}}\left|\left\langle\Omega\left|\mathcal{L}_{\text {odd }}\right| \pi^{0}(\mathbf{0})\right\rangle\right|_{m_{q}}^{\text {cont }}=0 .
$$

The meaning of this condition is rather simple. It amounts to fix, for each $k \geq 0$, the order $a^{2 k+1}$ contribution in the critical mass counter-term, $-M_{\mathrm{cr}}^{o p t} \bar{\psi}^{L} i \gamma_{5} \tau_{3} \psi^{L}$, in such a way that its vacuum to one-pion state matrix element compensates, in the limit of vanishing quark mass, the similar matrix element of the sum of all the other operators making up $\ell_{4+2 k+1}$.

In the next section we present concrete procedures designed to implement the condition (3.2) in actual simulations. To avoid confusion with the values the lattice action parameters will take in the successive steps of a simulation, we will provisionally put a bar over the symbols representing the values of the quark and the corresponding pion state mass while we develop the argument for the "optimal" determination of the critical mass.

\subsubsection{Lattice estimate}

We want to show how eq. (3.2), which has to do with a matrix element defined in the continuum theory, can be translated into a lattice condition. To this end let us consider the lattice correlator

$$
\left.a^{3} \sum_{\mathbf{x}}\langle\mathcal{Q}(x, 0)\rangle\right|_{\bar{m}_{q}} ^{L}=\left.a^{3} \sum_{\mathbf{x}}\left\langle V_{0}^{2}(x) P^{1}(0)\right\rangle\right|_{\bar{m}_{q}} ^{L}, \quad x_{0} \neq 0
$$


where $V_{0}^{2}=\bar{\psi} \gamma_{0} \frac{\tau_{2}}{2} \psi$ is the vector current with iso-spin index 2 and $P^{1}=$ $\bar{\psi} \gamma_{5} \frac{\tau_{1}}{2} \psi$ is the pseudo-scalar quark density with iso-spin index 1 . In the continuum the correlator (3.3) owing to parity vanishes for any value of $\bar{m}_{q}$, and we have

$$
\left.\left\langle Q_{V}^{2} P^{1}(0)\right\rangle\right|_{\bar{m}_{q}} ^{\text {cont }}=0
$$

where $Q_{V}^{2}=\int d \mathbf{x} V_{0}^{2}(\mathbf{x}, t)$ is the iso-spin 2 vector charge. On the lattice the breaking of parity (and iso-spin) due to the presence of the twisted Wilson term makes the correlator (3.3) non-vanishing by pure discretization effects. Extending to parity violating correlators the arguments developed in Sect. 2.1, one gets for its Symanzik expansion

$$
\begin{aligned}
& \left.a^{3} \sum_{\mathbf{x}}\langle\mathcal{Q}(x, 0)\rangle\right|_{\bar{m}_{q}} ^{L}= \\
& =\int d \mathbf{x}\left\{\left.\left\langle\Delta_{\text {odd }} \mathcal{Q}(x, 0)\right\rangle\right|_{\bar{m}_{q}} ^{\mathrm{cont}}-\left.\left\langle\mathcal{Q}(x, 0) \int d^{4} y \mathcal{L}_{\text {odd }}(y)\right\rangle\right|_{\bar{m}_{q}} ^{\mathrm{cont}}+\ldots,\right.
\end{aligned}
$$

where dots represent terms with higher order insertions of $\int d^{4} y \mathcal{L}_{\text {odd }}(y)$ and/or $\int d^{4} y \mathcal{L}_{\text {even }}(y)$ and $\Delta_{\text {odd }} \mathcal{Q}$ has the expression

$$
\begin{aligned}
& \Delta_{\text {odd }} \mathcal{Q}(x, 0)= \\
& =a\left[\eta \partial_{0} \bar{\psi} \gamma_{5} \frac{\tau_{1}}{2} \psi(x) \bar{\psi} \gamma_{5} \frac{\tau_{1}}{2} \psi(0)+\tilde{\eta} \bar{m}_{q} \bar{\psi} \gamma_{0} \gamma_{5} \frac{\tau_{1}}{2} \psi(x) \bar{\psi} \gamma_{5} \frac{\tau_{1}}{2} \psi(0)\right]+\mathrm{O}\left(a^{3}\right),
\end{aligned}
$$

In eq. (3.6) $\eta$ and $\tilde{\eta}$ are appropriate dimensionless coefficients, odd in $r$. We recall that in the language of the Symanzik improvement program the term we have explicitly written down in eq. (3.6) is the standard operator necessary for the on-shell $\mathrm{O}(a)$ improvement of $\mathcal{Q}(x, 0)$. In writing the expansion (3.5) use has been made of the continuum relation (3.4).

For the rest of the argument it is important to remark that from higher order insertions of $\int d^{4} y \mathcal{L}_{\text {odd }}(y)$ arbitrarily high powers of the ratio $\xi_{\pi}\left(\bar{m}_{q}\right) / \bar{m}_{\pi}^{2}$ will be generated in the r.h.s. of eq. (3.5)

At large times $(t \gg 1 / \Delta m$, where $\Delta m$ is the difference between the mass of the first excited state with the pion quantum numbers and that of the pion), we may write more explicitly the r.h.s. of eq. (3.5) in the form

$$
\begin{aligned}
& \left.\lim _{t \rightarrow+\infty}\left[\frac{e^{-\bar{m}_{\pi} t}}{2 \bar{m}_{\pi}}\right]^{-1} a^{3} \sum_{\mathbf{x}}\langle\mathcal{Q}(x, 0)\rangle\right|_{\bar{m}_{q}} ^{L}=\left[a \bar{m}_{q} \tilde{\eta}\left\langle\Omega\left|A_{0}^{1}\right| \pi^{1}(\mathbf{0})\right\rangle C_{S S L}+\right. \\
& \left.-a \bar{m}_{\pi} \eta\left\langle\Omega\left|P^{1}\right| \pi^{1}(\mathbf{0})\right\rangle C_{S L}-\frac{\xi_{\pi}\left(\bar{m}_{q}\right)}{2 \bar{m}_{\pi}^{2}} 2 \bar{m}_{\pi} C_{L}\right]\left.\left\langle\pi^{1}(\mathbf{0})\left|P^{1}\right| \Omega\right\rangle\right|_{\bar{m}_{q}} ^{\mathrm{cont}}
\end{aligned}
$$

with all corrections of order $a^{3}$ or higher encoded in the coefficient factors

$$
C_{X}=1+\left.\sum_{j, k, \ell=1}^{\infty} c_{j, k, \ell}^{(X)}\left(\frac{\xi_{\pi}\left(\bar{m}_{q}\right)}{\bar{m}_{\pi}^{2}}\right)^{j}\left(\frac{a^{2}}{\bar{m}_{\pi}^{2}}\right)^{k} a^{\ell}\right|_{j+\ell=\text { even }}, \quad X=L, S L, S S L .
$$


Several observations about the r.h.s. of eq. (3.7) are in order here. 1) Since $\left.\bar{m}_{q}\left\langle\Omega\left|A_{0}^{1}\right| \pi^{1}(\mathbf{0})\right\rangle\right|_{\bar{m}_{q}} ^{\text {cont }}$ is a quantity of order $\bar{m}_{\pi}^{3}$, the first term is completely immaterial to the present analysis. 2) The factor $\bar{m}_{\pi}$ in front of the second term comes from the time derivative in eq. (3.6). 3) The factor $2 \bar{m}_{\pi}$ in the third term arises from the chain of relations $\left.\left\langle\pi^{0}(\mathbf{0})\left|Q_{V}^{2}\right| \pi^{1}(\mathbf{q})\right\rangle\right|_{\bar{m}_{q}} ^{\text {cont }}=$ $\left.\left\langle\pi^{0}(\mathbf{0}) \mid \pi^{0}(\mathbf{q})\right\rangle\right|_{\bar{m}_{q}} ^{\text {cont }}=\left.(2 \pi)^{3} 2 \bar{m}_{\pi}\right|_{\bar{m}_{q}} ^{\text {cont }} \delta^{3}(\mathbf{q})$. 4) Concerning the factors $C_{L}, C_{S L}$ and $C_{S S L}$, they differ from unit by three different types of lattice artifacts. i) The terms with $k=\ell=0$ in $C_{L}$ represent the the leading "IR divergent" corrections contributing to the correlator $\left.a^{3} \sum_{\mathbf{x}}\langle\mathcal{Q}(x, 0)\rangle\right|_{\bar{m}_{q}} ^{L}$. ii) Sub-leading "IR divergent" corrections are generated by insertions of $\int d^{4} y \mathcal{L}_{\text {even }}(y)$ and/or pairs of $\int d^{4} y \mathcal{L}_{\text {odd }}(y)$ factors. They contribute extra $a^{2} / \bar{m}_{\pi}^{2}$ powers. iii) Finally there are "IR finite" corrections stemming, among others, from contributions of intermediate states other than zero three-momentum pions.

With all these premises, in order to fix the critical mass so as to have in the continuum (see eq. (3.2) and the definition (2.10) )

$$
\lim _{\bar{m}_{q} \rightarrow 0^{+}} \xi_{\pi}\left(\bar{m}_{q}\right)=0,
$$

one may think of proceeding on the lattice in the following way.

Given a first estimate of the critical mass, say $M_{\mathrm{cr}}^{e}$, consider the lattice action (2.1) where the critical mass has been momentarily replaced by the expression $M_{\mathrm{cr}}^{e}+\delta \tilde{m}$. In order to implement eq. (3.9) we must compute the lattice quantity in the l.h.s. of eq. (3.7) and identify the optimal value of the critical mass, $M_{\mathrm{cr}}^{o p t}$, as the limiting value of $M_{\mathrm{cr}}^{e}+\delta \tilde{m}$ at which the $\mathrm{O}\left(a^{0}\right)$ quantity (see eqs. (3.3) and (3.7))

$$
A\left(\bar{m}_{q}, M_{\mathrm{cr}}^{e}+\delta \tilde{m} ; t\right) \equiv\left[\frac{\bar{m}_{\pi}^{2}}{a} e^{\bar{m}_{\pi} t} a^{3} \sum_{\mathbf{x}}\langle\mathcal{Q}(x, 0)\rangle\right]_{\bar{m}_{q}}^{L}, \quad t \gg \frac{1}{\Delta m}
$$

vanishes as $\bar{m}_{q}$ is extrapolated to smaller and smaller values from the region where $\bar{m}_{q}>a$.

Numerically there can be various ways to do this. One possible strategy is to start from a value, $\bar{m}_{q}^{(1)}$, of the quark mass such that the order of magnitude inequality $\left(\bar{m}_{\pi}^{(1)}\right)^{2}>a$ holds. A first determination, $\delta \tilde{m}^{(1)}$, of $\delta \tilde{m}$ can be obtained by enforcing at large $t$ the condition

$$
A^{(1)} \equiv A\left(\bar{m}_{q}^{(1)}, M_{\mathrm{cr}}^{e}+\delta \tilde{m}^{(1)} ; t\right)=0 \quad t \gg \frac{1}{\Delta m},
$$

which in turn yields a first estimate, $\xi_{\pi}^{(1)}$, of the matrix element in (3.9). Solving iteratively the non-linear equation (3.11), one can write from eqs. (3.7) and (3.8)

$$
\xi_{\pi}^{(1)}=-a\left(\bar{m}_{\pi}^{(1)}\right)^{2} \eta\left\langle\Omega\left|P^{1}\right| \pi^{1}(\mathbf{0})\right\rangle+\delta \xi_{\pi}^{(1)},
$$


where the first term is the solution of the linearized form of (3.11) obtained by setting to unit the factors $C_{L}, C_{S L}$ and $C_{S S L}$ in eq. (3.7). The second term, $\delta \xi_{\pi}^{(1)}$, is the correction due to all the other terms and, in particular, to the higher powers of $\xi_{\pi}$. To estimate the magnitude of $\delta \xi_{\pi}^{(1)}$ we plug back the ansatz (3.12) in (3.11). The structure of the result is

$$
\delta \xi_{\pi}^{(1)}=a^{3} \sum_{k=0}^{\infty} h_{k}\left(\frac{a^{2}}{\left(\bar{m}_{\pi}^{(1)}\right)^{2}}\right)^{k}\left[1+\mathrm{O}\left(\left(\bar{m}_{\pi}^{(1)}\right)^{2}\right)+\mathrm{O}\left(a^{2}\right)\right] .
$$

where the leading $\mathrm{O}\left(a^{3}\right)$ corrections can be seen to arise from the terms with $k=1, j=\ell=0$ in eq. (3.8) ${ }^{3}$. The important point about eq. (3.13) is that $\delta \xi_{\pi}^{(1)}$ can be considered small under a condition, $\bar{m}_{q}^{(1)}>a^{2}$, which is weaker than the one $\left(\bar{m}_{q}^{(1)}>a\right)$ we have been using in establishing this result.

At this point one continues by lowering the quark mass to $\bar{m}_{q}^{(2)}<\bar{m}_{q}^{(1)}$, seeking for the new value, $\delta \tilde{m}^{(2)}$, of the mass shift which makes $A^{(2)}$ vanishing. The search must be performed in the neighborhoods of $\delta \tilde{m}^{(1)}$ to be sure that one remains in a region of the $\left(\bar{m}_{q}, M_{\mathrm{cr}}^{e}+\delta \tilde{m}\right)$-plane, where $\xi_{\pi} / \bar{m}_{\pi}^{2}$ is small, so that higher powers of the ratio $\xi_{\pi} / \bar{m}_{\pi}^{2}$ are even smaller. Proceeding in this way the convergence of the Symanzik expansion (3.7) is not put in danger as $\bar{m}_{q}$ is decreased. Rather, the initial convergence bound $\bar{m}_{q}>a$ is progressively weakened towards $\bar{m}_{q}>a^{2}$, as signaled by eq. (3.13).

A sequence, $\delta \tilde{m}^{(i)}$, of mass shifts is thus determined. If desired, these values can be numerically extrapolated to $\bar{m}_{q} \rightarrow 0$. The limiting value, $\delta \tilde{m}^{(\infty)}$, obtained in this way will allow to identify the "optimal" critical mass as the quantity

$$
M_{\mathrm{cr}}^{o p t}=M_{\mathrm{cr}}^{e}+\delta \tilde{m}^{(\infty)} .
$$

Notice that in all this procedure no lattice data points are employed where the bound $\bar{m}_{q}>a^{2}$ is violated. This caution is necessary because for $\bar{m}_{q} \leq$ $a^{2}$ large cutoff effects, which are hinted at by uncanceled non-leading "IR divergent" terms in the Symanzik expansion, cannot in general be excluded and reliable simulations may even be impossible because of metastabilities, if e.g. the peculiar lattice phase structure known as the "Sharpe-Singleton scenario" [16] is realized.

The method discussed above may seem unpractical in view of the fact that, especially for unquenched simulations, producing data at several values of the bare quark mass, as it is necessary to do if one wants to extrapolate to $\bar{m}_{q} \rightarrow 0$, is computationally rather demanding. We immediately notice,

\footnotetext{
${ }^{3}$ Actually only terms from $C_{L}$ and $C_{S L}$ are important to this order. Terms coming from $C_{S S L}$ are, in fact, negligible because of the extra $\bar{m}_{q}$ factor in front of the corresponding matrix element.
} 
however, that to all practical purposes (see the detailed argument given in Sect. 3.2.2 below) one can avoid such an extrapolation and just work with the "quasi-optimal" critical mass

$$
M_{\mathrm{cr}}^{q o p t}\left(\bar{m}_{q}^{\min }\right) \equiv M_{\mathrm{cr}}^{e}+\left.\delta \tilde{m}\right|_{\bar{m}_{q}^{\min }},
$$

where $\bar{m}_{q}^{\min }>a^{2}$ is the smallest of the bare quark masses of interest at the lattice spacing one is working and $\left.\delta \tilde{m}\right|_{\bar{m}_{q}^{\min }}$ is the solution of the equation $A\left(\bar{m}_{q}^{\min }, M_{\mathrm{cr}}^{e}+\left.\delta \tilde{m}\right|_{\bar{m}_{q}^{\min }} ; t\right)=0$.

The idea of estimating the critical mass at the smallest available value of $m_{q}$ was already put forward in the analysis of tm-LQCD performed in $\chi \mathrm{PT}$ in refs. 8, 9] and directly used in the unquenched simulations of ref. [15]. In quenched simulations slightly different versions of the strategy described above have been implemented in order to evaluate the (quasi-)optimal value of the critical mass. They all turned out to be quite effective in reducing cutoff artifacts [12, 13, 14].

\subsubsection{Left-over "IR divergent" cutoff effects}

To complete our analysis we have to determine what is the order of magnitude of the left-over discretization errors that will affect simulations carried out at non-vanishing $m_{q}$, when either i) eq. (3.14) or ii) eq. (3.15) for the critical mass is inserted in the Mtm-LQCD action (2.1). We will see that in both cases the situation will be very much like the one we encountered in Sect. 3.1. where we discussed the case in which the clover term was added to the MtmLQCD action.

i) Let us denote by the superscript "opt" lattice quantities computed using in the fermionic action the optimal critical mass, $M_{\mathrm{cr}}^{\text {opt }}$ (eq. (3.14)). The formerly leading $2 k$-fold pion pole contribution (2.9) now have an expression where $\xi_{\pi}\left(m_{q}\right)$ is systematically replaced by $\xi_{\pi}^{o p t}\left(m_{q}\right)$, which represents the value of the continuum matrix element $\left|\left\langle\Omega\left|\mathcal{L}_{\text {odd }}^{\text {opt }}\right| \pi^{0}(\mathbf{0})\right\rangle\right|$ at the current value, $m_{q}$, of the quark mass, with $\mathcal{L}_{\text {odd }}^{\text {opt }}$ the parity odd part of the Symanzik LEEA when the optimal critical mass is employed.

Although non-zero at non-vanishing quark mass, we want to show that $\xi_{\pi}^{o p t}\left(m_{q}\right) / m_{\pi}^{2}=\mathrm{O}(a)$. To this end we notice that one can write

$$
\xi_{\pi}^{o p t}\left(m_{q}\right)=\xi_{\pi}^{o p t}(0)+\left.\frac{\partial \xi_{\pi}^{o p t}\left(m_{q}\right)}{\partial m_{q}}\right|_{m_{q}=0} m_{q}+\ldots
$$

where from eqs. (3.12) and (3.13) one has $\xi_{\pi}^{\text {opt }}(0)=\mathrm{O}\left(a^{3}\right)$. This result comes from having extrapolated to $\bar{m}_{q}=0$ from the region $\bar{m}_{q}>a^{2}$, where $\delta \xi_{\pi}=\mathrm{O}\left(a^{3}\right)$. Since $\partial \xi_{\pi}^{o p t}\left(m_{q}\right) /\left.\partial m_{q}\right|_{m_{q}=0} \sim \mathrm{O}(a)$, we conclude that 
$\xi_{\pi}^{o p t}\left(m_{q}\right)$ is reduced to a mere order $a m_{q}$ quantity, implying, as announced, $\xi_{\pi}^{o p t}\left(m_{q}\right) / m_{\pi}^{2}=\mathrm{O}(a)$.

ii) If we set instead $M_{\mathrm{cr}}^{e}=M_{\mathrm{cr}}^{q o p t}\left(\bar{m}_{q}^{\min }\right)$ (eq. (3.15)) in the action (2.1), we get (notation should be self-explanatory)

$$
\xi_{\pi}^{q o p t}\left(m_{q}\right)=\xi_{\pi}^{q o p t}\left(\bar{m}_{q}^{\min }\right)+\left.\frac{\partial \xi_{\pi}^{q o p t}\left(m_{q}\right)}{\partial m_{q}}\right|_{\bar{m}_{q}^{\min }}\left(m_{q}-\bar{m}_{q}^{\min }\right)+\ldots
$$

where from eqs. (3.12) and (3.13) one finds $\xi_{\pi}^{\text {opt }}\left(\bar{m}_{q}^{\min }\right)=\mathrm{O}\left(a \bar{m}_{q}^{\min }\right)$, provided $\bar{m}_{q}^{\text {min }}>a^{2}$. From $\partial \xi_{\pi}^{q o p t}\left(m_{q}\right) /\left.\partial m_{q}\right|_{\bar{m}_{q}^{\min }} \sim \mathrm{O}(a)$ one obtains for any $m_{q} \geq \bar{m}_{q}^{\text {min }}$ the estimate $\xi_{\pi}^{q o p t}\left(m_{q}\right) / m_{\pi}^{2}=\mathrm{O}\left(a, a \bar{m}_{q}^{\min } / m_{q}\right)=\mathrm{O}(a)$.

The conclusion of this analysis is that both with the optimal as well as the quasi-optimal critical mass, the formerly leading "IR divergent" cutoff discretization errors are reduced to finite $\mathrm{O}\left(a^{2 k}\right)$ contributions.

This does not mean that all the non-leading "IR divergent" cutoff effects, which are of order $a^{2 k} /\left(m_{\pi}^{2}\right)^{h}, 2 k>h \geq 1$ ( $k, h$ integers), have disappeared from correlators. Actually by a non-trivial diagrammatic analysis, based on the structure of the non-leading "IR divergent" cutoff contributions, one can prove that the most "IR divergent" lattice artifacts left behind after using either the optimal or the quasi-optimal definition of the critical mass (eq. (3.14) or eq. (3.15)) are reduced down to order $a^{2}\left(a^{2} / m_{\pi}^{2}\right)^{k-1}(k \geq 1)$ effects at worse, just like in the case the clover term is employed. Notice, however, that in the case where the quasi-optimal critical mass is adopted this result holds only for $m_{q} \geq \bar{m}_{q}^{\min }$.

\section{The lattice GMOR relation}

In the previous sections we have shown how the leading "IR divergent" discretization effects can all be eliminated from the Symanzik expansion of lattice correlators. The left-over "IR divergent" terms of the expansion turn out to have the structure $a^{2} \sum_{\ell \geq 1} c_{\ell}\left(a^{2} / m_{q}\right)^{\ell}$. The convergence of this series sets the order of magnitude inequality $a^{2}<m_{\pi}^{2} \sim m_{q}$ from which one should determine the minimal value of the quark mass that (at fixed value of $a$ ) can be safely simulated before possibly encountering large discretization errors.

A workable way to numerically estimate this minimal value can be obtained by considering the behaviour of the charged pion mass as a function of $m_{q}$. It turns out, in fact, that in Mtm-LQCD there are Ward-Takahashi identities (WTI's) which take exactly the form they have in the formal continuum theory. From them a lattice GMOR relation can be derived.

To see how this works we recall that in Mtm-LQCD the 1-point split axial currents, $\hat{A}_{\mu}^{b}$, with iso-spin index $b=1,2$ are exactly conserved in the chiral 
limit $m_{q}=0$ [1, 2]. This implies the validity of the lattice WTI's

$$
\begin{gathered}
\left.\left\langle\left[\partial_{\mu}^{*} \hat{A}_{\mu}^{ \pm}(x)-2 m_{q} P^{ \pm}(x)\right] P^{\mp}(0)\right\rangle\right|_{m_{q}} ^{L}=\left.\left\langle S^{0}(0)\right\rangle\right|_{m_{q}} ^{L} \delta_{x, 0}, \\
\hat{A}_{\mu}^{ \pm}=\hat{A}_{\mu}^{1} \pm i \hat{A}_{\mu}^{2}, \quad P^{ \pm}=\bar{\psi}^{L} \gamma_{5} \frac{\tau_{1} \pm i \tau_{2}}{2} \psi^{L}, \quad S^{0}=\bar{\psi}^{L} \psi^{L}
\end{gathered}
$$

After integration over space-time, one gets for any $m_{q} \neq 0$

$$
\left.2 m_{q} a^{4} \sum_{x}\left\langle P^{ \pm}(x) P^{\mp}(0)\right\rangle\right|_{m_{q}} ^{L}=-\left.\left\langle S^{0}(0)\right\rangle\right|_{m_{q}} ^{L} .
$$

Although, as the WTI (4.3) itself shows, there is no mixing between $S^{0}$ and the identity operator with a cubically (or a linearly) divergent coefficient, there is still room for a quadratically divergent term proportional to $m_{q}$. Indeed, the l.h.s. of eq. (4.3) is equal to the piece where intermediate states are inserted plus a divergent contribution of the kind $m_{q} / a^{2}$ coming from the (integrated) short-distance singularity of the correlator $\left\langle P^{ \pm}(x) P^{\mp}(0)\right\rangle$ at $x=0$. This term should be brought to the r.h.s. of the equation, thus leaving finite (subtracted) expressions in both sides of the resulting equation.

We can now repeat on the lattice the argument that in the continuum leads to the classical GMOR relation, if we assume that spontaneous chiral symmetry breaking occurs in the limiting continuum theory, i.e. if we assume that

$$
\Sigma \equiv-\left.\lim _{m_{q} \rightarrow 0}\left\langle\Omega\left|S^{0}\right| \Omega\right\rangle\right|_{m_{q}} ^{\text {cont }} \neq 0
$$

As in the formal continuum theory, we insert a complete set of states in the subtracted correlator in the l.h.s. of eq. (4.3). We obtain in this way

$$
\left.m_{\pi^{ \pm}}^{2}\right|_{m_{q}} ^{L}=\left.2 m_{q} \frac{\left|\left\langle\Omega\left|P^{ \pm}\right| \pi^{ \pm}\right\rangle\right|^{2}}{\left[-\left\langle\Omega\left|S_{\mathrm{sub}}^{0}\right| \Omega\right\rangle\right]}\right|_{m_{q}} ^{L}+\ldots
$$

where we have explicitely written down only the contribution coming from the pion pole. Dots are terms due to the intermediate states that stay massive as $m_{q} \rightarrow 0$ as well as terms vanishing with $m_{q}$ faster than linearly (the latter include in particular $\mathrm{O}\left(a^{2} m_{q}^{2}\right)$ terms stemming from cutoff effects in the sum over $\left.x_{0}{ }^{4}\right)$. The subscript "sub" is to remind us that it is the properly subtracted chiral condensate (or, more precisely, the vacuum expectation

\footnotetext{
${ }^{4} \mathrm{No} \mathrm{O}(a)$ terms can arise from the sum over $x_{0}$ in the l.h.s. of eq. (4.3), as in Mtm-LQCD symmetry arguments ensure that only $\mathrm{O}\left(a^{2}\right)$ discretization errors can affect expectation values of parity even operators.
} 
value of the properly subtracted iso-singlet scalar density operator) that enters this equation. Notice that, as expected, the r.h.s. of eq. (4.5) is a finite renormalization group invariant quantity in the limit $a \rightarrow 0$.

Once the leading "IR divergent" cutoff effects have been canceled out, the use of the Symanzik expansion in the r.h.s. of eq. (4.5) yields the formula

$$
\left.m_{\pi^{ \pm}}^{2}\right|_{m_{q}} ^{L}=\left.2 m_{q} \frac{\left|\left\langle\Omega\left|P^{ \pm}\right| \pi^{ \pm}\right\rangle\right|^{2}}{\Sigma}\right|_{m_{q}=0} ^{\text {cont }}\left[1+a^{2} \sum_{\ell \geq 0} b_{\ell}\left(\frac{a^{2}}{m_{q}}\right)^{\ell}\right]+\ldots
$$

where dots denote less dangerous "IR divergent" lattice artifacts compared to those explicitely shown as well as contributions of higher order in $m_{q}$. In getting eq. (4.6) we have used the fact that the continuum limit of $-\left\langle\Omega\left|S_{\text {sub }}^{0}\right| \Omega\right\rangle^{L}$ at vanishing quark mass is $\Sigma \neq 0$ (see eq. (4.4)).

From the above analysis it follows that, in the region where the series in eq. (4.6) converges (i.e. at least where the order of magnitude inequality $m_{\pi}^{2} \sim m_{q}>a^{2}$ is satisfied), the squared mass of the charged lattice pion is linear in $m_{q}$ (up to small $\mathrm{O}\left(a^{4}\right)$ and $\mathrm{O}\left(m_{q}^{2}\right)$ effects). Thus, vice-versa, we can imagine to use deviations from the established linear behaviour possibly seen at small $m_{q}$ as a workable criterion to determine the minimal value of $m_{q}$ at which simulations can be performed before being set-off by discretization effects.

\section{$5 \quad$ Hadron masses and pion decay constant}

We wish to discuss in this section some peculiar issues concerning the magnitude of the $\mathrm{O}\left(a^{2}\right)$ discretization artifacts affecting hadronic energies (in particular masses) and the pion decay constant.

\subsection{Hadron energies}

As a consequence of the special form of the diagrams contributing to energies (self-energy diagrams), one can get convinced that the latter are less "IR divergent" than the correlators from which they can be extracted, in the sense that at fixed order in $a$, the most "IR divergent" lattice corrections to their continuum limit contain one overall factor $1 / m_{\pi}^{2}$ less than the "IR divergent" cutoff effects appearing in correlators. The reason is that at least one among the inserted $\int \mathcal{L}_{\text {odd }}$ operators gets absorbed in a multi-particle matrix element, with the consequence that it is not anymore available for producing a pion pole 
An explicit calculation shows that for the difference between lattice and continuum energy of the hadron $\alpha_{n}$

$$
\Delta E_{\alpha_{n}}(\mathbf{q}) \equiv \frac{1}{2}\left[E_{\alpha_{n}}^{L}(\mathbf{q})+E_{\alpha_{n}}^{L}(-\mathbf{q})\right]-E_{\alpha_{n}}^{\mathrm{cont}}(\mathbf{q})
$$

one gets at order $a^{2}$ the estimate

$$
\begin{aligned}
& \left.\Delta E_{\alpha_{n}}(\mathbf{q})\right|_{a^{2}} \propto \\
& \left.\propto \frac{a^{2}}{m_{\pi}^{2}} \operatorname{Re} \frac{\left\langle\Omega\left|\ell_{5}\right| \pi^{0}(\mathbf{0})\right\rangle\left\langle\pi^{0}(\mathbf{0}) \alpha_{n}(\mathbf{q})\left|\ell_{5}\right| \alpha_{n}(\mathbf{q})\right\rangle_{\text {conn }}}{2 E_{\alpha_{n}}(\mathbf{q})}\left[1+\mathrm{O}\left(m_{\pi}^{2}\right)\right]\right|_{m_{q}} ^{\mathrm{cont}}
\end{aligned}
$$

where the subscript conn denotes the completely connected part of the matrix element $\left\langle\pi^{0}(\mathbf{0}) \alpha_{n}(\mathbf{q})\left|\ell_{5}\right| \alpha_{n}(\mathbf{q})\right\rangle$. In eq. (5.2) the "IR divergent" $\mathrm{O}\left(a^{2} / m_{\pi}^{2}\right)$ piece comes from the continuum correlator with two $\mathcal{L}_{\text {odd }}=a \ell_{5}+\mathrm{O}\left(a^{3}\right)$ insertions. The latter gives also rise to $\mathrm{O}\left(a^{2}\right)$ "IR finite" corrections. A further $\mathrm{O}\left(a^{2}\right)$ "IR finite" correction comes from a single insertion of $\mathcal{L}_{\text {even }}=a^{2} \ell_{6}+$ $\mathrm{O}\left(a^{4}\right)$ and contributes a term proportional to $a^{2}\left\langle\alpha_{n}(\mathbf{q})\left|\ell_{6}\right| \alpha_{n}(\mathbf{q})\right\rangle / 2 E_{\alpha_{n}}(\mathbf{q})$.

It should be noted that the "IR divergent" lattice artifact in $\left.\Delta E_{\alpha_{n}}\right|_{a^{2}}$ is reduced to an "IR finite" correction after anyone of the two "cures" described in Sect. 3 .

Specializing the formula (5.2) to the case of pions, one obtains the interesting result that the difference between charged and neutral pion (square) masses is a finite $\mathrm{O}\left(a^{2}\right)$ quantity even if the critical mass has not been set to its "optimal" value or the clover term has not been introduced. The reason is that the leading "IR divergent" contributions shown in eq. (5.2) are equal for all pions (as one can prove by standard soft pion theorems [19]), hence cancel in the (square) mass difference. This conclusion is in agreement with results from $\chi \mathrm{PT}[16$.

\subsection{Pion decay constant}

Data on a (quenched) computation of $f_{\pi}$, carried out in Mtm-LQCD using the value of the critical mass obtained from the vanishing of the pion mass, show cutoff effects that are seen to deviate at small bare quark masses from the straight line extrapolation drawn from large masses [4]. This behaviour (called "bending phenomenon" in ref. 4]) sets in at values of $m_{q}$ around $a \Lambda_{\mathrm{QCD}}^{2}, \Lambda_{\mathrm{QCD}} \sim 200 \mathrm{MeV}$. Furthermore the detailed recent scaling test of [14] indicates that the "bending phenomenon" is an $\mathrm{O}\left(a^{2}\right)$ deviation with a magnitude which increases as $m_{\pi^{ \pm}}^{2}$ is lowered. 
In the works of refs. [4, 12, 13, 14, the lattice pion decay constant, $f_{\pi}^{L}$, was extracted from the formula [2]

$$
f_{\pi}^{L}\left(m_{\pi^{ \pm}}^{2}\right)=\left.2 m_{q} \frac{\left\langle\Omega\left|P^{ \pm}\right| \pi^{\mp}\right\rangle}{m_{\pi^{ \pm}}^{2}}\right|_{m_{q}} ^{L},
$$

from which it is seen that $f_{\pi}^{L}$ is a ratio of two lattice quantities.

It should be noted that in general the matrix element $\left.\left\langle\Omega\left|P^{ \pm}\right| \pi^{\mp}\right\rangle\right|_{m_{q}} ^{L}$ in the numerator is affected by leading "IR divergent" cutoff effects which however, like in the case of hadron masses, are multiplied by an extra factor of $m_{\pi}^{2} \sim$ $m_{q}$. This peculiar property can be traced back to the invariance of the correlator $\left.\left\langle P^{ \pm}(x) P^{\mp}(y)\right\rangle\right|_{m_{q}} ^{L}$ (from which the matrix element $\left.\left\langle\Omega\left|P^{ \pm}\right| \pi^{\mp}\right\rangle\right|_{m_{q}} ^{L}$ is extracted) under axial rotations around the third iso-spin direction. At the price of shifting the bare quark mass from $m_{q}$ to $\widetilde{m}_{q}=m_{q}+\mathrm{O}\left(a^{2} / m_{q}\right)$ one can, in fact, always think of having performed the axial rotation with an angle such to bring the critical mass to its optimal value. A Symanzik expansion of $\left.\left\langle P^{ \pm}(x) P^{\mp}(y)\right\rangle\right|_{\tilde{m}_{q}} ^{L}$ evaluated with the optimal critical mass then shows that the leading "IR divergent" cutoff effects (which from this viewpoint arise owing to $\widetilde{m}_{q} \neq m_{q}$ only) are softened by the extra multiplicative $m_{\pi}^{2}$ factor. Similarly it follows from the discussion of Sect. 5.1 (which is consistent with the previous argument) that also $\left.m_{\pi^{ \pm}}^{2}\right|_{m_{q}} ^{L}$ is affected by leading "IR divergent" cutoff artifacts softened by an overall $m_{\pi}^{2}$ factor. The latter, however, drops when the relative lattice correction is considered.

Inserting in eq. (5.3) the behaviour of numerator and denominator we have just discussed, we find that $f_{\pi}^{L}$ is in general affected by "IR divergent" relative corrections that are fully leading in our nomenclature, i.e. of the kind $\left(a / m_{\pi}^{2}\right)^{2 k}, k \geq 1$. Of course, if the appropriate clover term or the "optimal" critical mass is introduced in the action, these "IR divergent" relative lattice artifacts are reduced to $a^{2}\left(a^{2} / m_{\pi}^{2}\right)^{k-1}, k \geq 1$ effects.

To be concrete at order $a^{2}$ one finds that in $f_{\pi}^{L}$ (see eq. (5.3)) relative corrections of the type $a^{2} / m_{q}^{2}$ are present, which come only from $\left.\left(m_{\pi^{ \pm}}^{2} / m_{q}\right)\right|_{m_{q}} ^{L}$. Less important corrections of the type $a^{2} / m_{q}$ and $a^{2}$ come instead from both $\left.\left\langle\Omega\left|P^{ \pm}\right| \pi^{\mp}\right\rangle\right|_{m_{q}} ^{L}$ and $\left.\left(m_{\pi^{ \pm}}^{2} / m_{q}\right)\right|_{m_{q}} ^{L}$. After the clover improvement or the "optimal" critical mass cure, the relative cutoff effects in $f_{\pi}^{L}$ get reduced to plain $a^{2}$ terms. Results consistent with these features have also been derived in $\chi \mathrm{PT}$ studies of Mtm-LQCD [8, 20, 5] at (next-to) leading order.

A beautiful confirmation of the validity of the analysis presented in this section comes from the fact that, when the critical mass is set at its "optimal" value (yielding $\xi_{\pi}^{\text {opt }}\left(m_{q}\right)=\mathrm{O}\left(a m_{\pi}^{2}\right)$ ), no "bending phenomenon" is anymore visible in the $f_{\pi}^{L}$ data, as demonstrated by the results of ref. [12, 13, 14] (see also [21]). 


\section{Conclusions}

We have shown in this paper that lattice correlators in Mtm-LQCD are affected by discretization artifacts that tend to become large when the quark mass is decreased. Cutoff effects of this kind can be appropriately described in terms of the Symanzik LEEA of the lattice theory, and turn out to arise from multiple pion poles associated with the insertions of the parity odd (and iso-spin non-invariant) piece of the effective action, $\int d^{4} y \mathcal{L}_{\text {odd }}(y)$.

At order $a^{2 k}$ the leading "IR divergent" cutoff effects (those with $h=2 k$ in eq. (1.1)) are associated to $2 k$-fold pion poles. Since their residues contain $2 k$ factors of the matrix element $\xi_{\pi}=\left|\left\langle\Omega\left|\mathcal{L}_{\text {odd }}\right| \pi^{0}(\mathbf{0})\right\rangle\right|=\left|a\left\langle\Omega\left|\ell_{5}\right| \pi^{0}(\mathbf{0})\right\rangle\right|+\mathrm{O}\left(a^{3}\right)$, all these dangerous contributions are eliminated from lattice correlators if we can let $\xi_{\pi}$ vanish sufficiently fast as the pion mass goes to zero. This can indeed be achieved if Mtm-LQCD is improved à la Symanzik by the inclusion of the lattice clover term in the action or by appropriately tuning the critical mass parameter to what we have called its "optimal" value.

In both cases the left-over "IR divergent" cutoff effects are at worse of order $a^{2}\left(a^{2} / m_{\pi}^{2}\right)^{k-1}, k \geq 1$.

The discussion we presented in this work does not make use of chiral perturbation theory and treats $m_{q}$ as a fixed quantity in the limit in which $a \rightarrow 0$. By using the notion of Symanzik LEEA, one is able to obtain results to all orders in $a$. It must be recalled, however, that, as recently pointed out in ref. [5, a $\chi \mathrm{PT}$ analysis of (M)tm-LQCD shows that it is possible to reabsorb (at any given order in the chiral power counting of choice) a whole tower of cutoff effects in a shift of the chiral lattice vacuum, thus extending the radius of convergence of the associated expansion ${ }^{5}$.

Our detailed findings are in agreement with the results of refs. [9, 8, [5] concerning the quark mass dependence of lattice artifacts and the existence of an "optimal" choice for the critical mass yielding a complete cancellation of the leading "IR divergent" cutoff effects. In particular, we agree with the authors of ref. [8] that, once the "optimal" value of the critical mass is employed in Mtm-LQCD simulations (or the clover term is introduced), the tight (order of magnitude) limitation $m_{q}>a$ on the quark mass is substantially weakened and brought down to the much more favourable bound $m_{q}>a^{2}$, beyond which large mass-dependent cutoff effects may show up in simulations at fixed $a$.

We conclude this paper with a remark which explains how the optimal critical mass prescription can be viewed as a peculiar mapping between bare and renormalized parameters and may be useful in practice to correct for a

\footnotetext{
${ }^{5}$ We are indebted to S. Sharpe and M. Golterman for valuable discussions on this point.
} 
not optimal choice of the critical mass. Indeed, the fact that it is possible to get rid of all the leading "IR divergent" cutoff effects by working at an optimal value of the critical mass implies that, even if simulations are carried out with a non-optimal choice of $M_{\mathrm{cr}}$, one can still effectively eliminate all the dangerous $\left(a / m_{q}\right)^{2 k}(k \geq 1)$ terms by means of a slightly more elaborate analysis of the available simulation data. This analysis consists in using an appropriately redefined expression of the renormalized quark mass (by $\mathrm{O}\left(a^{2}\right)$ terms), accompanied by a small $(\mathrm{O}(a))$ chiral rotation in the third iso-spin direction of all quantities that have non-trivial chiral transformation properties ${ }^{6}$. By construction the lattice correlators that are obtained in this way will have a Symanzik expansion where the leading "IR-divergent" cutoff effects are just absent, while automatic $\mathrm{O}(a)$ improvement is obviously preserved (the deviations from the initial simulation values of the critical mass and twist angle are in fact $\mathrm{O}(a)$ quantities). However this analysis requires some knowledge (or a simultaneous determination, which can be obtained by working at several values of $\left(M_{0}, m_{q}\right)$ ) of scale-independent combinations of the renormalization constants of operators belonging to the same chiral multiplet, such as $Z_{P} / Z_{S}=Z_{P} Z_{m}$ or $Z_{V} / Z_{A}$.

Indeed the prescription we have described is the microscopic counter-part of what is suggested in ref. [8] at the level of the effective chiral theory in order to reabsorb the contributions of these lattice artifacts via a proper redefinition of the vacuum state.

Being based on the assumption that an acceptable (i.e. $\mathrm{O}(a)$ accurate) estimate of the critical mass is already known, this remark is of no use to obtain such an estimate (the argument would become circuitous). It should also be observed that the effort of determining by how much the renormalized quark mass have to be shifted and chiral operators rotated is essentially equivalent (modulo the knowledge of $Z_{P} / Z_{S}$ and analogous renormalization constant ratios) to enforce the optimality condition in the way we have described it in sect. 3.2

Acknowledgments - We are grateful to the anonymous referee for his valuable observations. We wish to thank K. Jansen, M. Lüscher, S. Sharpe and M. Testa for useful discussions and V. Lubicz and M. Golterman for correspondence. We are grateful to K. Jansen, M. Papinutto, A. Shindler, C. Urbach and I. Wetzorke for information about the results of ref. [13] prior to publication. One of us (GCR) wishes to thank the Humboldt Foundation

\footnotetext{
${ }^{6}$ This is the kind of procedure we have alluded to in sect. 5.2 below eq. (5.3). Detailed formulae for this analysis can be obtained on the basis of the results in ref. [2] about the relation between bare and renormalized mass parameters and currents (or more general operators) in tmLQCD.
} 
for financial support.

\section{Appendix - $\mathrm{O}\left(a^{2 k+1}\right)$ improvement of Mtm-LQCD}

We prove in this Appendix the absence of all the terms of order $a^{2 k+1}, k \geq 0$, in the Symanzik expansion of the expectation value of parity-even (multilocal) gauge-invariant and multiplicatively renormalizable operators in MtmLQCD. Here to be precise by "parity-even" we mean an operator which goes exactly into itself under parity. This result follows from the symmetry $P \times \mathcal{D}_{d} \times\left(m_{q} \rightarrow-m_{q}\right)$, enjoyed by the lattice action, where $\left(x_{P}=(-\mathbf{x}, t)\right)$

$$
\mathcal{P}:\left\{\begin{array}{l}
U_{0}(x) \rightarrow U_{0}\left(x_{P}\right), \\
U_{k}(x) \rightarrow U_{k}^{\dagger}\left(x_{P}-a \hat{k}\right), \quad k=1,2,3 \\
\psi(x) \rightarrow \gamma_{0} \psi\left(x_{P}\right) \\
\bar{\psi}(x) \rightarrow \bar{\psi}\left(x_{P}\right) \gamma_{0}
\end{array}\right.
$$

is the physical parity of the theory, and

$$
\mathcal{D}_{d}: \begin{cases}U_{\mu}(x) & \rightarrow U_{\mu}^{\dagger}(-x-a \hat{\mu}), \quad \mu=0,1,2,3 \\ \psi(x) & \rightarrow e^{3 i \pi / 2} \psi(-x) \\ \bar{\psi}(x) & \rightarrow e^{3 i \pi / 2} \bar{\psi}(-x)\end{cases}
$$

The proof of $\mathrm{O}\left(a^{2 k+1}\right)$ improvement of Mtm-LQCD follows immediately from the observation that necessarily the Symanzik LEEA which describes the lattice artifacts of Mtm-LQCD is invariant under (the continuum version of) the transformation $P \times \mathcal{D}_{d} \times\left(m_{q} \rightarrow-m_{q}\right)$. This invariance implies, in particular, that all the terms of order $a^{2 k+1}, k \geq 0$, in (2.2) are odd under parity. In fact, $\mathcal{D}_{d} \times\left(m_{q} \rightarrow-m_{q}\right)$ counts the parity of the overall dimension, $d$, of any product of elementary fields and mass factors, by multiplying it by the phase factor $\exp (i \pi d)$ (besides inverting its space-time argument). As a result, all the continuum correlators in the Symanzik expansion that are multiplied by an odd power of $a$ necessarily correspond to expectation values of parity odd operators. Since parity is an exact continuum symmetry, they all vanish.

Few comments are in order here.

1) It is interesting to observe that automatic $\mathrm{O}\left(a^{2 k+1}\right), k \geq 0$, improvement (in the Symanzik sense) is a very robust property of Mtm-LQCD. Being based on straight symmetry arguments, it is independent of any consideration on possible phase transition scenarios [9, 16. In fact, for any value of $m_{q}$, sufficiently close to the continuum limit the chiral phase of the vacuum will be finally determined by the quark mass term in the action and not by the Wilson term. 
2) At maximal twist the $r$-parity properties of the critical mass are immaterial to the above argument for automatic $\mathrm{O}\left(a^{2 k+1}\right), k \geq 0$, improvement. The reason is that in this situation (but not for generic values of the twist angle) the Wilson term, and thus necessarily also its critical mass counter-term, are odd under parity. This clearly remains true for whatever determination of the critical mass is taken, independently of its behaviour under $r \rightarrow-r$.

On the contrary, at non-maximal twist, the proof of $\mathrm{O}(a)$ improvement (via Wilson averaging) requires the critical mass to be an odd function of $r$. As explained in the papers of ref. [2], this condition can (and should) always be imposed when determining $M_{\mathrm{cr}}(r)$.

3) Finally we note that the conclusions about automatic $\mathrm{O}\left(a^{2 k+1}\right)$ improvement reached in this Appendix can be extended to the case of maximally twisted quarks with non-degenerate masses [3]. If the physical basis of ref. [3] is employed, a proof completely analogous to that given above for mass degenerate quarks can be given relying now on the symmetry $P \times \mathcal{D}_{d} \times\left(m_{q} \rightarrow\right.$ $\left.-m_{q}\right) \times\left(\epsilon_{q} \rightarrow-\epsilon_{q}\right)$, with $\epsilon_{q}$ the bare mass splitting within the quark pair.

\section{References}

[1] R. Frezzotti, P.A. Grassi, S. Sint and P. Weisz, Nucl. Phys. B (Proc. Suppl.) 83 (2000) 941 and JHEP 0108 (2001) 058;

R. Frezzotti, S. Sint and P. Weisz [ALPHA Collaboration], JHEP 0107 (2001) 048 (hep-lat/0104014);

M. Della Morte, R. Frezzotti, J. Heitger and S. Sint [ALPHA Collaboration], JHEP 0110 (2001) 041;

R. Frezzotti and S. Sint, Nucl. Phys. B (Proc. Suppl.) 106 (2002) 814.

[2] R. Frezzotti and G.C. Rossi, JHEP 0408 (2004) 007 and Nucl. Phys. B (Proc. Suppl.) 129 (2004) 880;

R. Frezzotti, Nucl. Phys. B (Proc. Suppl.) $140 \quad$ (2005) 134 (hep-lat/0409138).

[3] R. Frezzotti and G.C. Rossi, Nucl. Phys. B (Proc. Suppl.) 128 (2004) 193 (hep-lat/0311008).

[4] W. Bietenholz et al. [ $\chi \mathrm{LF}$ Collaboration], JHEP 0412 (2004) 044 (hep-lat/0411001).

[5] S.R. Sharpe, Phys. Rev. D72 (2005) 074510 (hep-lat/0509009).

[6] B. Sheikholeslami and R. Wohlert, Nucl. Phys. B259 (1985) 572.

[7] M. Lüscher, S. Sint, R. Sommer and P. Weisz, Nucl. Phys. B478 (1996) 365; 
M. Lüscher, S. Sint, R. Sommer, P. Weisz and U. Wolff, Nucl. Phys. B491 (1997) 323.

[8] S.R. Sharpe and J.M.S. Wu, Phys. Rev. D71 (2005) 074501 (hep-lat/0411021).

[9] S. Aoki and O. Bär, Phys. Rev. D70 (2004) 116011 (hep-lat/0409006).

[10] M. Gell-Mann, R.J. Oakes and B. Renner, Phys. Rev. 175 (1968) 2195.

[11] K. Jansen, A. Shindler, C. Urbach and I. Wetzorke [ $\chi$ LF Collaboration], Phys. Lett. B586 (2004) 432, (hep-lat/0312013);

A. Shindler, K. Jansen, C. Urbach and I. Wetzorke, Nucl. Phys. B (Proc. Suppl.) 140 (2005) 746.

[12] A.M. Abdel-Rehim, R. Lewis and R.M. Woloshyn, Phys. Rev. D71 (2005) 094505 (hep-lat/0503007).

[13] K. Jansen, M. Papinutto, A. Shindler, C. Urbach and I. Wetzorke $[\chi$ LF Collaboration], Phys. Lett. B619 (2005) 184 (hep-lat/0503031).

[14] K. Jansen, M. Papinutto, A. Shindler, C. Urbach and I. Wetzorke $[\chi$ LF Collaboration], JHEP 0509 (2005) 071 (hep-lat/0507010);

K. Jansen, M. Papinutto, A. Shindler, I. Wetzorke, C. Urbach, PoS LAT2005:231,2005 (hep-lat/0509194).

[15] F. Farchioni, R. Frezzotti, K. Jansen, I. Montvay, G.C. Rossi, E. Scholz, A. Shindler, N. Ukita, C. Urbach, I. Werzorke, Eur. Phys. J. C 39 (2005) 421 (hep-lat/0406039) and Nucl. Phys. B (Proc. Suppl.) 140 (2005) 240 (hep-lat/0409098);

F. Farchioni, K. Jansen, I. Montvay, E. Scholz, L. Scorzato, A. Shindler, N. Ukita, C. Urbach and I. Wetzorke, Eur. Phys. J. C42 (2005) 73 (hep-lat/0410031);

F. Farchioni, K. Jansen, I. Montvay, E.E. Scholz, L. Scorzato, A. Shindler, N. Ukita, C. Urbach, U. Wenger, I. Wetzorke, Phys. Lett. B624 (2005) 324 (hep-lat/0506025);

F. Farchioni et al., PoS LAT2005:072,2005 (hep-lat/0509131).

[16] S. Aoki, Phys. Rev. D30 (1984) 2653 and Phys. Rev. Lett. 57 (1986) 3136

S.R. Sharpe and R. Singleton, Jr., Phys. Rev. D58 (1998) 074501 (hep-lat/9804028);

G. Münster, JHEP 0409 (2004) 035 (hep-lat/0407006);

L. Scorzato, Eur. Phys. J. C 37 (2004) 445 (hep-lat/0407023);

S.R. Sharpe and J.M.S. Wu, Phys. Rev. D 70 (2004) 094029 (hep-lat/0407025). 
[17] G. Heatlie, G. Martinelli, C. Pittori, G.C. Rossi and C.T. Sachrajda, Nucl. Phys. B352 (1991) 266.

[18] See e.g. S. Weinberg, "The Quantum Theory of Fields", Cambridge University Press (Cambridge, 1998), Vol. 1, sect. 10.2.

[19] S. Weinberg, Phys. Rev. D7 (1973) 1068.

[20] S. Aoki and O. Bär, PoS LAT2005:046,2005 (hep-lat/0509002).

[21] A. Shindler, PoS LAT2005:014,2005 (hep-lat/0511002). 